\title{
ENUMERATION OF SMALL NONISOMORPHIC 1-ROTATIONAL TWOFOLD TRIPLE SYSTEMS
}

\author{
YEOW MENG CHEE AND GORDON F. ROYLE
}

\begin{abstract}
In this paper, twofold triple systems of order $v$ are enumerated for all $v \leq 19$.
\end{abstract}

\section{INTRODUCTION}

The existence of $\operatorname{TS}(v, 2)$ 's (all terms are defined in $\S 2)$ is completely settled; the condition $v \equiv 0$ or $1(\bmod 3)$ is known to be both necessary and sufficient [4]. On the other hand, enumeration efforts have not enjoyed such success. In fact, the exact number of pairwise nonisomorphic $\mathrm{TS}(v, 2)$ 's, denoted $N(v)$, has been determined only for $v \leq 10$. In particular, we have $N(3)=N(4)=1$ (trivial), $N(6)=1[5], N(7)=4[13], N(9)=36[12,8]$, and $N(10)=960$ $[1,3]$. One reason for the unavailability of such enumeration results for higher values of $v$ is the inherent computational complexity of the problem that leads to a combinatorial explosion effect. To curb this combinatorial explosion, extra conditions are often imposed to enumerate interesting classes of designs. One such condition involves specifying automorphisms that the desired designs must possess.

Cyclic $\operatorname{TS}(v, 2)$ 's, that is, $\operatorname{TS}(v, 2)$ 's possessing an automorphism of order $v$, have been enumerated by Colbourn [2] for $v \leq 16$. In this paper, we completely enumerate the class of 1-rotational $\operatorname{TS}(v, 2)$ 's for all $v \leq 19$. The existence of 1-rotational $\operatorname{TS}(v, 2)$ 's is determined by Kuriki and Jimbo [7], who proved that the necessary condition $v \equiv 0$ or $1(\bmod 3)$ is also sufficient. However, they did not provide any enumeration results. The only result on the enumeration of 1-rotational $t-(v, k, \lambda)$ designs for $\lambda>1$ that we are aware of is that by Mathon and Rosa [9], who determined that there are precisely 85 nonisomorphic 1-rotational 2-(15, 5, 4) designs.

\section{DEFINITIONS AND NOTATIONS}

A $t$-design, or more specifically $t-(v, k, \lambda)$ design, is a combinatorial system consisting of a pair $(X, \mathscr{B})$, where $X$ is a finite set of $v$ elements called points, and $\mathscr{B}$ is a collection of $k$-subsets of $X$ called blocks, such that every $t$-subset of $X$ is contained in precisely $\lambda$ blocks. We generally allow $t$-designs to have repeated blocks. A 2- $(v, 3, \lambda)$ design is commonly called a $\lambda$-fold triple system of order $v$, and is denoted $\operatorname{TS}(v, \lambda)$.

Received by the editor June 29, 1990.

1991 Mathematics Subject Classification. Primary 05B05. 
Two $t$-designs, say $\left(X_{1}, \mathscr{B}_{1}\right)$ and $\left(X_{2}, \mathscr{B}_{2}\right)$, are said to be isomorphic if there exists a bijection $\pi: X_{1} \rightarrow X_{2}$ such that $\left\{x_{1}, x_{2}, \ldots, x_{k}\right\} \in \mathscr{B}_{1}$ if and only if $\left\{\pi\left(x_{1}\right), \pi\left(x_{2}\right), \ldots, \pi\left(x_{k}\right)\right\} \in \mathscr{B}_{2}$. Such a bijection is called an isomorphism from $\left(X_{1}, \mathscr{B}_{1}\right)$ onto $\left(X_{2}, \mathscr{B}_{2}\right)$. An automorphism of a $t$-design $(X, \mathscr{B})$ is an isomorphism from $(X, \mathscr{B})$ onto itself. The set of all automorphisms of a $t$-design forms a group, called the full automorphism group of the design, under functional composition. Any subgroup of the full automorphism group is simply called an automorphism group.

A $t$-design is called 1-rotational if it admits a permutation with one fixed point and a cycle of length $v-1$ as an automorphism.

\section{Computational details}

Let $G$ be a group acting on a finite set $X$ of $v$ points. Then there is a natural action of $G$ on the 2-subsets and 3-subsets of $X$. Let $A(G)$ be a matrix with its rows and columns indexed by $G$-orbits of 2 -subsets and 3subsets of $X$, respectively, and define the $(i, j)$ th entry of $A(G), a_{i j}$, as the number of 3-subsets in the $G$-orbit indexing column $j$ that contain a fixed orbit representative of the $G$-orbit indexing row $i$. The number $a_{i j}$ is independent of the choice of the orbit representative. A more general result of Kramer and Mesner [6] implies that a $\operatorname{TS}(v, 2)$ exists with $G$ as an automorphism group if and only if there exists a nonnegative integral vector $u$ satisfying the matrix equation $A(G) u=2 j$, where $j$ is the vector of all 1 's. It should be clear that the vector $u$ determines which orbits of 3-subsets (or blocks) are to be present in the $\operatorname{TS}(v, 2)$ in a natural way.

Let $G=\langle\alpha\rangle$, where $\alpha=(0)(12 \cdots v-1)$. It follows from a previous discussion that if we want to construct a 1-rotational $\operatorname{TS}(v, 2)$ on the set of points $X=\{0,1, \ldots, v-1\}$, we need only to look for a nonnegative integral vector $u$ such that $A(G) u=2 j$. We can compute the size of the matrix $A(G)$, that is, the number of orbits of 2-subsets and 3-subsets of $X$ under the action of $G$, from the Cauchy-Frobenius-Burnside lemma:

Lemma 1. Let $G$ be a group acting on a finite set $X$, and let $\chi(\pi)$ be the set of all $t$-subsets of $X$ that is invariant under the permutation $\pi \in G$. Then the number of $G$-orbits of $t$-subsets of $X$ is

$$
\frac{1}{|G|} \sum_{\pi \in G}|\chi(\pi)| .
$$

Table 1 provides information on the size of the $A(G)$ matrices for various values of $v$.

A branch-and-bound algorithm using depth-first search was used to find all nonnegative integral vectors $u$ that satisfy $A(G) u=2 j$. These resulted in a set of distinct designs which are then subjected to further analysis to remove isomorphic copies. Isomorphism testing of the designs was carried out using nauty, the isomorphism checking algorithm of McKay [10,11]. Let $N_{1 r}(v)$ and $N_{1 r}^{*}(v)$ denote the number of pairwise nonisomorphic 1-rotational $\operatorname{TS}(v, 2)$ 's without and with repeated blocks, respectively. A summary of the enumeration results we obtained is given in Table 2 .

All the pairwise nonisomorphic 1-rotational $\operatorname{TS}(v, 2)$ 's for $v \leq 19$ are listed in the Appendix to be found on the microfiche card attached to this issue. The blocks in each $\operatorname{TS}(v, 2)$ can be obtained by developing the given starter blocks 


\begin{tabular}{rc}
\multicolumn{2}{c}{ TABLE 1} \\
\hline$v$ & size of $A(G)$ \\
\hline 6 & $3 \times 4$ \\
7 & $4 \times 7$ \\
9 & $5 \times 11$ \\
10 & $5 \times 14$ \\
12 & $6 \times 20$ \\
13 & $7 \times 25$ \\
15 & $8 \times 33$ \\
16 & $8 \times 38$ \\
18 & $9 \times 48$ \\
19 & $10 \times 55$ \\
\hline
\end{tabular}

TABLE 2. Nonisomorphic $\operatorname{TS}(v, 2)$ 's

\begin{tabular}{rrrr}
\hline$v$ & $N_{1 r}(v)$ & $N_{1 r}^{*}(v)$ & Total \\
\hline 6 & 1 & 0 & 1 \\
7 & 1 & 1 & 2 \\
9 & 1 & 2 & 3 \\
10 & 1 & 0 & 1 \\
12 & 5 & 0 & 5 \\
13 & 8 & 5 & 13 \\
15 & 36 & 17 & 53 \\
16 & 48 & 5 & 53 \\
18 & 174 & 12 & 186 \\
19 & 366 & 99 & 465 \\
\hline
\end{tabular}

of each design with the permutation $(0)(12 \cdots v-1)$. We also give the order of the full automorphism group of each design.

\section{TRANSITIVE AND AfFine DESIGNS}

A $\operatorname{TS}(v, 2)(X, \mathscr{B})$ is transitive if its full automorphism group acts transitively on the set of points $X$. Let $X$ be the set of elements underlying the 
finite field $\operatorname{GF}(v)$, where $v$ is a prime power. Then a $\operatorname{TS}(v, 2)(X, \mathscr{B})$ is said to be affine if it admits the affine group

$$
\{x \mapsto a x+b \mid a \neq 0 ; a, b \in \mathrm{GF}(v)\}
$$

as an automorphism group. It is not difficult to see that an affine $\operatorname{TS}(v, 2)$ is both transitive and 1-rotational.

It appears that transitive $\operatorname{TS}(v, 2)$ 's are relatively rare among the 1-rotational $\operatorname{TS}(v, 2)$ 's. In all the pairwise nonisomorphic $\operatorname{TS}(v, 2)$ 's enumerated for $v \leq$ 19 , only six transitive $\operatorname{TS}(v, 2)$ 's were found. These designs are a $\operatorname{TS}(6,2)$ (design \#1), a $\operatorname{TS}(7,2)$ (design \#1), $\mathrm{TS}(9,2)$ (design \#3), a $\mathrm{TS}(13,2)$ (design \#2), a $\operatorname{TS}(16,2)$ (design \#45), and a $\operatorname{TS}(19,2)$ (design \#189). Further analysis reveals that all of these designs are actually affine designs, except for the $\operatorname{TS}(6,2)$. It also follows that the number of pairwise nonisomorphic affine $\operatorname{TS}(v, 2)$ 's is precisely one for each $v \in\{7,9,13,16,19\}$. We note that the affine $\operatorname{TS}(7,2), \operatorname{TS}(9,2), \operatorname{TS}(13,2)$, and $\operatorname{TS}(16,2)$ must have been known to Colbourn [2] too, since every affine $\operatorname{TS}(v, 2)$ is also cyclic.

\section{BIBLIOGRAPHY}

1. C. J. Colbourn, M. J. Colbourn, J. J. Harms, and A. Rosa, A complete census of $(10,3,2)$ block designs and of Mendelsohn triple systems of order ten, iii. (10, 3, 2) block designs without repeated blocks, Congr. Numer. 39 (1983), 211-234.

2. M. J. Colbourn, Cyclic block designs: computational aspects of their construction and analysis, Ph.D. Thesis, Department of Computer Science, University of Toronto, Toronto, Ontario, 1980.

3. B. Ganter, A. Gulzow, R. Mathon, and A. Rosa, A complete census of $(10,3,2)$-block designs and of Mendelsohn triple systems of order ten, iv. (10, 3, 2)-block designs with repeated blocks, Math. Schriften Kassel 5/78.

4. H. Hanani, The existence and construction of balanced incomplete block designs, Ann. Math. Statist. 32 (1961), 361-386.

5. M. Hall, Jr., Combinatorial theory, Blaisdell, Waltham, MA, 1967.

6. E. S. Kramer and D. M. Mesner, t-designs on hypergraphs, Discrete Math. 15 (1976), 263296.

7. S. Kuriki and M. Jimbo, On 1-rotational $S_{\lambda}(2,3, v)$ designs, Discrete Math. 46 (1983), 33-40.

8. R. Mathon and A. Rosa, A census of Mendelsohn triple systems of order nine, Ars Combin. 4 (1977), 309-315.

9.

10. B. D. McKay, Practical graph isomorphism, Congr. Numer. 30 (1981), 45-87.

11. __ Nauty user's guide, Computer Science Technical Report TR-CS-84-05, Australian National University, 1984.

12. E. J. Morgan, Some small quasi-multiple designs, Ars Combin. 3 (1977), 233-250.

13. H. K. Nandi, Enumeration of nonisomorphic solutions of balanced incomplete block designs, Sankhyā 7 (1946), 305-312.

Planning and Infrastructure Department, National Computer Board, 71 Science PARK DRIVE, S0511, RePUblic OF Singapore

E-mail address: yeowmeng@iti.gov.sg

Department of Computer Science, University of Western Australia, Nedlands 6009, Western Australia, Australia

E-mail address: gordon@cs.uwa.oz.au 